\title{
Stimulation of rat cranial dura mater with potassium chloride causes CGRP release into the cerebrospinal fluid and increases medullary blood flow
}

\author{
Mária Dux ${ }^{\mathrm{a}}$, Christine Will ${ }^{\mathrm{b}}$, Mirjam Eberhardt ${ }^{\mathrm{c}}$, Michael J.M. Fischer ${ }^{\mathrm{b}}$, Karl Messlinger ${ }^{\mathrm{b}, *}$ \\ a Department of Physiology, University of Szeged. Dóm tér 10, H-6720 Szeged, Hungary \\ ${ }^{\mathrm{b}}$ Institute of Physiology and Pathophysiology, Friedrich-Alexander University Erlangen-Nürnberg, Universitätsstrasse 17, D-91054 Erlangen, Germany \\ c Department of Anaesthesia and Critical Care Medicine, Hannover Medical School, Carl-Neuberg-Str. 1, 30625 Hannover, Germany
}

\section{A R T I C L E I N F O}

\section{Article history:}

Received 6 September 2016

Received in revised form 30 December 2016

Accepted 8 February 2017

Available online $\mathrm{xxxx}$

\section{Keywords:}

Meningeal afferents

Neuropeptides

Jugular plasma

Laser Doppler flowmetry

Headache

\begin{abstract}
A B S T R A C T
Primary headaches may be accompanied by increased intracranial blood flow induced by the release of the potent vasodilator calcitonin gene-related peptide (CGRP) from activated meningeal afferents. We aimed to record meningeal and medullary blood flow simultaneously and to localize the sites of CGRP release in rodent preparations in vivo and ex vivo.

Blood flow in the exposed rat parietal dura mater and the medulla oblongata was recorded by laser Doppler flowmetry, while the dura was stimulated by topical application of $60 \mathrm{mM}$ potassium chloride $(\mathrm{KCl})$. Samples of jugular venous plasma and cerebrospinal fluid (CSF) collected from the cisterna magna were analysed for CGRP concentrations using an enzyme immunoassay. In a hemisected rat skull preparation lined with dura mater the CGRP releasing effect of $\mathrm{KCl}$ superfusion was examined.

Superfusion of the dura mater with $\mathrm{KCl}$ decreased meningeal blood flow unless alpha-adrenoceptors were blocked by phentolamine, whereas the medullary blood flow was increased. The same treatment caused increased CGRP concentrations in jugular plasma and CSF and induced significant CGRP release in the hemisected rat skull preparation. Anaesthesia of the trigeminal ganglion by injection of lidocaine reduced increases in medullary blood flow and CGRP concentration in the CSF upon meningeal $\mathrm{KCl}$ application.

CGRP release evoked by depolarisation of meningeal afferents is accompanied by increased blood flow in the medulla oblongata but not the dura mater. This discrepancy can be explained by the smooth muscle depolarising effect of $\mathrm{KCl}$ and the activation of sympathetic vasoconstrictor mechanisms. The medullary blood flow response is most likely mediated by CGRP released from activated central terminals of trigeminal afferents. Increased blood supply of the medulla oblongata and CGRP release into the CSF may also occur in headaches accompanying vigorous activation of meningeal afferents.
\end{abstract}

(c) 2017 Elsevier Ltd. All rights reserved.

\section{Introduction}

The cranial dura mater and cerebral arteries are innervated by unmyelinated and thinly myelinated afferent nerve fibers arising from the trigeminal ganglion (Andres et al., 1987; Steiger and Meakin, 1984). An exclusively nociceptive function is attributed to these afferents, based on classical intraoperative studies showing that stimulation of meningeal structures evokes no other sensation than headaches (Feindel et al., 1960; Ray and Wolff, 1940). A considerable proportion of meningeal afferents contains neuropeptides like calcitonin gene-related peptide (CGRP) (Keller and Marfurt, 1991; Messlinger et al., 1995; O'Connor and van der Kooy, 1988) and CGRP release is an established method to quantify meningeal afferent activation in vitro

* Corresponding author at: Institute of Physiology and Pathophysiology, University of Erlangen-Nürnberg, Universitätsstr. 17, D-91054 Erlangen, Germany.

E-mail address: karl.messlinger@fau.de (K. Messlinger). and in vivo (Bellamy et al., 2006; Ebersberger et al., 1999; Goadsby et al., 1988). CGRP is a potent vasodilator of intracranial arteries inducing increases in meningeal blood flow (Escott et al., 1995; Jansen-Olesen et al., 1996; Messlinger et al., 1995). Thus recordings of meningeal vasodilatation and blood flow have been used to examine the effects of chemical mediators presumably involved in meningeal nociception (Akerman et al., 2002; Dux et al., 2002; Escott et al., 1995) and to test substances designed for headache therapy (Messlinger et al., 1997; Petersen et al., 2005; Tröltzsch et al., 2007). CGRP can also be released from the trigeminal ganglion (Eberhardt et al., 2009) and from central terminals of trigeminal afferents within the spinal trigeminal nucleus (Amrutkar et al., 2011; Jansen-Olesen et al., 2014; Kageneck et al., 2014; Offenhauser et al., 2005), where it acts most likely as pro-nociceptive mediator (Meng et al., 2009; Storer et al., 2004).

Although CGRP has been attributed a key role in primary headaches for many years (Arulmani et al., 2004a; Edvinsson and Goadsby, 1994; Goadsby and Edvinsson, 1994; Lassen et al., 2002), its nociceptive 
function remains elusive. Increases in CGRP plasma levels have been found in acute phases of migraine and cluster headache (Bellamy et al., 2006; Goadsby et al., 1988, 1990) and also interictally in chronic migraine (Cernuda-Morollón et al., 2013). Inhibition of CGRP receptors is clearly therapeutic in migraine (Ho et al., 2008; Olesen et al., 2004) and inactivation of CGRP or CGRP receptors by four newly developed humanized monoclonal antibodies is promising in reducing the frequency of headache attacks in chronic and frequent migraine based on a variety of clinical phase-3 studies (Bigal et al., 2015; Dux and Messlinger, 2015; Giamberardino et al., 2016; Schuster and Rapoport, 2016).

The lack of understanding the role of CGRP in migraine pain may be partly due to our ignorance of its precise sites of release and action. While there is no doubt that CGRP is released when meningeal afferents are massively activated, it is unclear whether the bulk of CGRP found in plasma samples is released at peripheral or central sites in animal experiments (Goadsby et al., 1988) as well as in patients during headache attacks (Bellamy et al., 2006; Goadsby et al., 1990). CGRP released from central afferent terminals or from afferents innervating pial blood vessels can be expected to accumulate in the cerebrospinal fluid (CFS) rather than in the venous compartment due to the blood-brain barrier. Finally, blood flow increase should also occur in the medullary dorsal area, when central trigeminal afferents are activated. Release of CGRP during nociceptive processing may thereby support the blood supply of central structures for an increased metabolism. Using video imaging, our group has recently shown that local electrical stimulation of the rat cranial dura mater causes vasodilatation of pial arteries of the dorsal medulla (Will et al., 2016).

The preparation described here extends our established models of meningeal nociception which employed CGRP release from meningeal afferents in the hemisected rodent skull preparation (Ebersberger et al., 1999; Fischer and Messlinger, 2007; Gupta et al., 2010), trigeminal ganglion (Eberhardt et al., 2008) and brainstem (Amrutkar et al., 2011; Kageneck et al., 2014) and meningeal blood flow recordings in the exposed cranial dura mater in rats (Dux and Messlinger, 2001; Dux et al., 2003; Kurosawa et al., 1995). The aim of the present study was to measure CGRP concentrations in samples of jugular plasma and CSF of the cisterna cerebello-medullaris (cisterna magna) together with changes in medullary blood flow provoked by chemical depolarization of meningeal afferents as a rat model of meningeal nociception (Fig. 1A). We hypothesized that massive activation of meningeal afferents, as it is assumed to occur in severe headaches, causes release of CGRP into plasma and CSF and is accompanied by an increase in blood flow not only in the cranial dura mater but also in pial blood vessels of the medulla oblongata.

\section{Methods}

\subsection{Experimental animals and surgery}

All experimental procedures were carried out in accordance with the ethical issues of the International Association for the Study of Pain and in compliance with the guidelines for the welfare of experimental animals of the Federal Republic of Germany and the European Union (Council Directive 2010/63/EU). The experimental protocols were reviewed by an ethics committee and approved by the local District Government. Efforts were made to keep the number of animals as low as possible. Adult male Wistar rats weighing 300-400 g (10-14 weeks old) were used. The animals were anaesthetized with $5 \%$ isoflurane (Forene, Abbott, Wiesbaden, Germany) inhaled in a closed box, continued by application of $2 \%$ isoflurane through a tight mask. In most experiments for plasma CGRP measurements (see below) animals were initially anaesthetized with intraperitoneal (i.p.) application of 120-

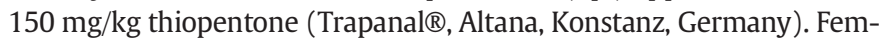
oral artery and vein were cannulated for recording of blood pressure and intravenous (i.v.) infusion of substances. In animals designated for

\section{A Experimental setup}

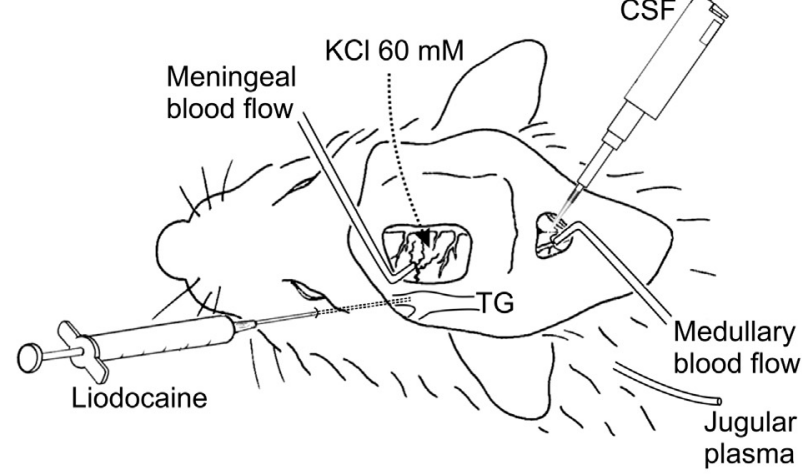

B Meningeal blood flow

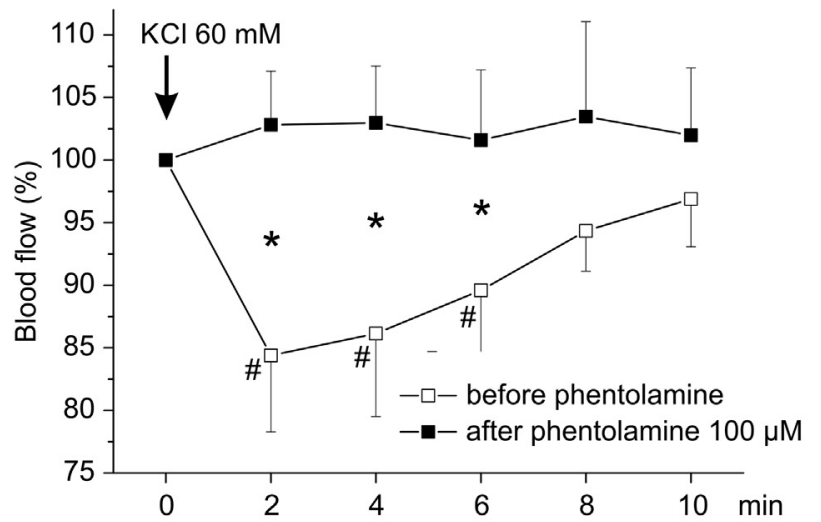

C Medullary blood flow

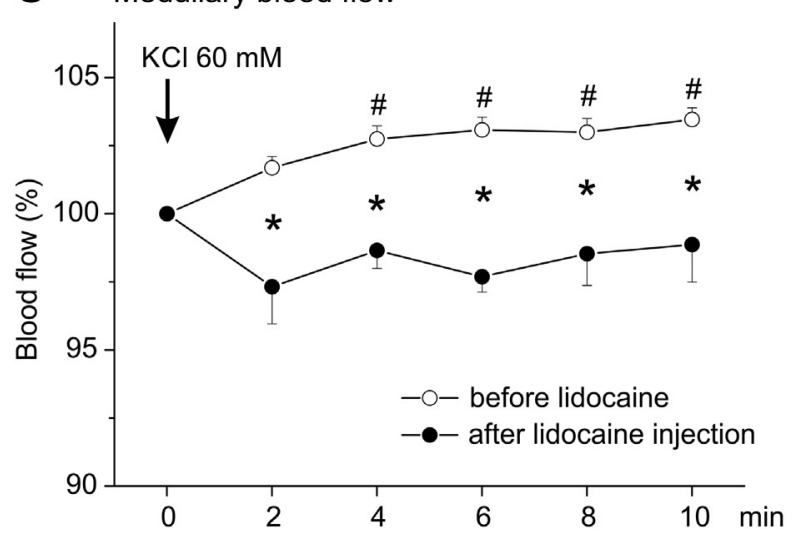

Fig. 1. Experimental setup (A) and changes in meningeal and medullary blood flow (normalized to baseline) during topical application of potassium chloride $(\mathrm{KCl} 60 \mathrm{mM}$ ) onto the dura mater (B, C). A: Laser Doppler L-type needle probes were positioned upon arterial vessels in the exposed dura mater and the medulla oblongata. Collection of cerebrospinal fluid (CSF) from the surface of the medulla and of jugular venous blood was combined with flow measurements and with injection of lidocaine into the trigeminal ganglion (TG) in some series of experiments. B: Response of dural blood vessels to $\mathrm{KCl}$ before and after phentolamine hydrochloride $(100 \mu \mathrm{M})$ measured at intervals of $2 \mathrm{~min}$; ${ }^{*}$ different to baseline (ANOVA), * different to the effect of $\mathrm{KCl}$ prior to blocking alpha-adrenoceptors ( $t$-test; $n=10)$. C: Effect of trigeminal ganglion anaesthesia on medullary blood flow induced by $\mathrm{KCl}(60 \mathrm{mM}) ;{ }^{*}$ different to baseline (ANOVA), ${ }^{*}$ different to the effect of $\mathrm{KCl}$ prior to ganglion anaesthesia ( $t$-test; $\mathrm{n}=10$ ).

venous blood sampling, a plastic catheter was introduced in cranial direction into the jugular vein of the side ipsilateral to the trepanation (see below) and flushed with saline containing $10 \mathrm{U} / \mathrm{ml}$ heparin (Ratiopharm, Ulm, Germany). The opening of the catheter was placed close to the bifurcation of the jugular vein. The animals were tracheotomized and artificially ventilated with a mixture of oxygen- 
enriched room air and $2 \%$ isoflurane. In most experiments for plasma CGRP measurements anaesthesia was maintained by small doses of thiopentone $(25 \mathrm{mg} / \mathrm{kg}$ i.p. or $10 \mathrm{mg} / \mathrm{kg}$ i.v.) when required. There was no obvious difference between the measurements in plasma obtained from animals anaesthetized with either of these procedures. Depth of anaesthesia was routinely assessed and held at a level in which noxious stimuli (pinching of earlobes) failed to elicit motor reflexes or changes in systemic arterial pressure. The body temperature of the animals was recorded with a thermoprobe inserted into the rectum and was kept at $37-37.5{ }^{\circ} \mathrm{C}$ with a feedback-controlled heating pad (TKM 0902, Föhr Medical Instruments, Seeheim, Germany). Systemic blood pressure was recorded with a pressure transducer connected to the catheter inserted into the right femoral artery. The expiratory $\mathrm{CO}_{2}$ was continuously monitored (Artema MM 200, Karl Heyer, Bad Ems, Germany) and maintained at 3-3.5\%.

The head of the animal was fixed in a stereotaxic frame and the skin overlying the skull and the neck region was opened. The neck muscles were divided along the midline and the medulla oblongata was exposed by cutting the atlanto-occipital ligament and the underlying dura mater (Fig. 1A). For dural blood flow recording a cranial window was drilled into the parietal bone, exposing the branches of the middle meningeal artery (Kurosawa et al., 1995). To avoid thermal lesions, a saline-cooled drill was used. For trigeminal ganglion anaesthesia a thin silicon tube connected to a 27-gauge needle was filled with fractions of $40 \mu \mathrm{l}$ of $2 \%$ lidocaine hydrochloride (Xylocain, AstraZeneca, Wedel, Germany) and $40 \mu \mathrm{l}$ of $0.1 \%$ methylene blue (Sigma, Taufkirchen, Germany) separated by an air bubble of $10 \mu$. The needle was introduced through the infraorbital canal into the ipsilateral trigeminal ganglion (Fig. 1A). At the end of the experiments, the animals were killed with an overdose of thiopentone ( $250 \mathrm{mg} / \mathrm{kg}$ i.v.).

\subsection{Measurement of medullary and dural blood flow and systemic arterial pressure}

The exposed dura mater in the cranial window was covered with $40 \mu$ of synthetic interstitial fluid (SIF) containing (in $\mathrm{mM}$ ): 107.8 $\mathrm{NaCl}, 26.2 \mathrm{NaHCO}_{3}, 9.64 \mathrm{Na}$-gluconate, 7.6 sucrose, 5.55 glucose, 3.5 $\mathrm{KCl}, 1.67 \mathrm{NaH}_{2} \mathrm{PO}_{4}, 1.53 \mathrm{CaCl}_{2}$ and $0.69 \mathrm{MgSO}_{4}$, buffered to $\mathrm{pH} 7.4$ with carbogen gas $\left(95 \% \mathrm{O}_{2}, 5 \% \mathrm{CO}_{2}\right)$. For stimulation with $\mathrm{KCl}$, the SIF was replaced by SIF containing $60 \mathrm{mM} \mathrm{KCl}$. Solutions were removed after $10 \mathrm{~min}$ and the dura mater was washed repeatedly with SIF to allow the blood flow to return to the basal level. In some experiments the nonselective alpha-adrenergic antagonist phentolamine hydrochloride at $100 \mu \mathrm{M}$ (Sigma, Taufkirchen, Germany) was applied to the dura mater for 5 min prior to stimulation with $60 \mathrm{mM} \mathrm{KCl}$.

Medullary and dural blood flow was recorded with needle L-type probes of a laser Doppler flowmeter (DRT4, Moor Instruments, Axminster, UK) positioned over the arterial blood vessels of the exposed dorsal surface of the medulla oblongata and branches of the middle meningeal artery supplying the dura mater (Fig. 1A). Blood flow was recorded at a sampling rate of $10 \mathrm{~Hz}$ and expressed in arbitrary units (AU). Medullary and meningeal blood flow and systemic blood pressure of the animals were recorded simultaneously. Data were stored and processed with the MoorSoft program for Windows. The basal blood flow (baseline) was the mean flow value measured during a 3 minute period prior to the stimulation of the dura mater. Percentage changes in meningeal and medullary blood flow in response to $\mathrm{KCl}$ application were determined as mean flow values within the $10 \mathrm{~min}$ application period calculated separately at two-minute intervals relative to the basal flow. The possible contribution of sympathetic mechanisms to the $\mathrm{KCl}$-induced vascular response was determined by comparing the changes in blood flow in response to $\mathrm{KCl}$ before and after topical application of the alpha-adrenergic antagonist phentolamine hydrochloride $(100 \mu \mathrm{M}$, Sigma, Taufkirchen, Germany). In parallel, the mean arterial blood pressure was registered during the whole stimulation period and compared to baseline.
After defining the effect of $\mathrm{KCl}$ on meningeal and medullary blood flow the trigeminal ganglion was anaesthetised by slowly injecting lidocaine through the catheter inserted into the ganglion. Two minutes later $60 \mathrm{mM} \mathrm{KCl}$ was applied to the dura mater for $10 \mathrm{~min}$ while the medullary blood flow was recorded. The effect of trigeminal ganglion anaesthesia on the $\mathrm{KCl}$-induced medullary blood flow was determined by comparing blood flow measured before and after lidocain injection into the ganglion. At the end of the experiment the correct position of the injection needle was controlled by injection of methylene blue and subsequent dissection of the trigeminal ganglion. Experiments in which the ganglion showed no blue staining were discarded.

\subsection{Measurement of CGRP concentration in plasma and cerebrospinal fluid}

Venous blood samples of $300 \mu$ were taken through the jugular catheter at intervals of $15 \mathrm{~min}$ with a $1 \mathrm{ml}$ syringe containing $10 \mu \mathrm{l}$ of $0.5 \mathrm{M}$ EDTA (Sigma, Taufkirchen, Germany) to prevent blood coagulation. Each blood sample was immediately transferred to Eppendorf cups and diluted with $60 \mu$ l enzyme-linked immunoassay (EIA) buffer (Bertin Pharma/SPIbio, Montigny le Bretonneux, France) containing peptidase inhibitors. The samples were centrifuged at $2500 \mathrm{rpm}$ for $2 \mathrm{~min}$, then the plasma supernatant was taken off with a pipette and stored at $-20{ }^{\circ} \mathrm{C}$ for later analysis. Care was taken not to cause hemolysis during each step of this procedure.

The CSF covering the exposed dorsal surface of the medulla oblongata was collected with a micropipette without touching the tissue. First the impact of the head surgery on the CGRP concentration in the CSF was studied. A first sample of $25 \mu \mathrm{lCSF}$ was collected immediately after opening the cisterna magna and a second 15 min later. After trepanation of the skull, three to six other CSF samples of $25 \mu \mathrm{l}$ were taken at intervals of $15 \mathrm{~min}$. In the main experiments starting 30 min after completing the surgery when the postpreparatory values of CGRP concentration in the CSF had reached a stable baseline level, the effects of $\mathrm{KCl}$ stimulation $(60 \mathrm{mM})$ were determined by comparing the CGRP concentrations of CSF samples taken $10 \mathrm{~min}$ before and immediately after stimulation of the dura mater. All samples were immediately transferred to Eppendorf cups and diluted with EIA buffer at a volume of 4 times the volume of CSF, then they were stored at $-20^{\circ} \mathrm{C}$ for later analysis.

The CGRP content of the samples was measured by EIA (Bertin Pharma/SPIbio, Montigny le Bretonneux, France). The absorbance of the reaction product representing the CGRP content of the sample was determined photometrically, using a microplate reader (Opsys MR, Dynex Technologies, Denkendorf, Germany). The lower limit of detection is $5 \mathrm{pg} / \mathrm{ml} \mathrm{CGRP}$ as communicated by the manufacturer. The plasma CGRP concentration was calculated in $\mathrm{pg} / \mathrm{ml}$. Changes induced in CGRP release by $\mathrm{KCl}$ were expressed as percentage changes relative to the basal release.

\subsection{Ex vivo measurements of meningeal CGRP release}

An ex vivo rat dura mater preparation was used to assess the release of CGRP from meningeal afferents. Rats were decapitated after lethal $\mathrm{CO}_{2}$ inhalation. The skull was divided into halves along the midline after the removal of the skin and the muscles. The cerebral hemispheres were removed, skull halves were washed at room temperature for $30 \mathrm{~min}$ in SIF, then placed in a humid chamber and the cranial fossae were filled with $400 \mu \mathrm{L}$ SIF. Samples of the superfusate were collected with a micropipette at periods of $5 \mathrm{~min}$ for CGRP measurement. Two control samples were taken in order to determine basal CGRP release then the dura was stimulated with $60 \mathrm{mM} \mathrm{KCl} .100 \mu \mathrm{l}$ of samples diluted with $25 \mu$ EIA buffer were placed into Eppendorf cups and immediately frozen at $-20{ }^{\circ} \mathrm{C}$ for subsequent analysis with EIA as described above. The CGRP concentrations of the superfusates were measured in $\mathrm{pg} / \mathrm{ml}$ and expressed as percentage changes relative to the basal release. 
2.5. CGRP- and tyrosine hydroxylase-immunhistochemistry of dura and pia mater

After the measurements of medullary and meningeal blood flow the animals were injected with an overdose of thiopentone. Immediately after cessation of spontaneous breathing but ongoing heart beat they were thoracotomized and perfused through the ventricle with $200 \mathrm{ml}$ saline followed by $200 \mathrm{ml}$ of $4 \%$ paraformaldehyde in phosphate buffer ( $\mathrm{pH}$ 7.4). The brain including dura mater and medulla was dissected and postfixed for $24 \mathrm{~h}$. Then the cranial dura mater and the medullary pia mater were carefully removed and processed as a whole mount for staining with the indirect immunofluorescence technique using a rabbit polyclonal antiserum raised against CGRP (1:100, Dianova, Hamburg, Germany) and (for the dura mater) a sheep polyclonal antiserum raised against tyrosine hydroxylase (1:2000, Novus Biologicals, Littleton, CO, USA). Donkey anti-rabbit or anti-sheep IgG (1:1000) labeled with Alexa 488 or Alexa 555 (Molecular Probes, Eugene, OR, USA) were used as secondary antibodies. Tissues were examined with a fluorescence microscope (Leica MZ FLIII, Leica Microsystems, Bensheim, Germany) equipped with a Leica DFC 320 digital camera system. Confocal images were taken with appropriate filter and pinhole settings using a $20 \times$ dry objective lens and sequential scanning. Single planes or stacks of images were merged into a $515 \times 512$ pixel RGB tiff-file using confocal software ZEN 2010. The final layout was made with CorelDraw (Corel, Dublin, Ireland).

\subsection{Statistics}

Sample sizes were determined using power calculations on the basis of previous experiments, from which the variance of changes in blood flow and CGRP release induced by meningeal stimulation were known. Pre- and poststimulation values were compared using the paired Student's $t$-test (for $n \geq 10$ ) or the Wilcoxon test (for $n<10$ ) and presented as means \pm SEM. ANOVA with repeated measurements and Fisher's least significant difference (LSD) test were used to analyze the consecutive measurements of CGRP levels at intervals of $15 \mathrm{~min}$ and the flow values at two-minute intervals. $P<0.05$ was considered statistically significant.

\section{Results}

\subsection{Stimulation-induced changes in meningeal blood flow}

The application of depolarizing $\mathrm{KCl}(60 \mathrm{mM})$ onto the dura mater in 10 experiments decreased the meningeal blood flow within 2 min by $15.6 \pm 6.1 \%$, which was significant up to the 6th min (repeated measures ANOVA and LSD-test, $p<0.001-0.05$ ) before it slowly recovered approximating the baseline towards the end of the 10-min application period (Fig. 1B). Five minutes after pretreatment of the dura mater with the alpha-adrenoceptor antagonist phentolamine, $\mathrm{KCl}$ application was followed by a tendency of increased meningeal blood flow, which was not significant within the 10 min application period compared to baseline (repeated measures ANOVA). The difference between the normalized flow values of the corresponding time intervals before and after phentolamine was significant up to the 6th min (two-way repeated measures ANOVA and LSD test, $p<0.001-0.05$ ) (Fig. 1B).

Injection of lidocaine into the ipsilateral trigeminal ganglion in 10 experiments did not cause significant changes in basal meningeal blood flow ( $t$-test). The subsequent application of $\mathrm{KCl}$ was not followed by a significant change in flow increase compared to the effect of $\mathrm{KCl}$ before ganglion anaesthesia (repeated measures ANOVA).

\subsection{Stimulation-induced changes in medullary blood flow and arterial} blood pressure

Application of $\mathrm{KCl}$ to the dura mater in 10 experiments induced a small but consistent increase in medullary blood flow from the 2nd two-min interval (ANOVA and LSD test, $p \leq 0.01$ ). The maximal increase by $3.6 \pm 0.5 \%$ occurred towards the end of the 10 -min application period (Fig. 1C).

Injection of lidocaine into the trigeminal ganglion in 10 experiments did not significantly change the basal medullary flow ( $t$-test). However, after lidocaine injection, meningeal $\mathrm{KCl}$ application did no longer cause significant changes in medullary blood flow (repeated measures ANOVA). The difference between the normalized flow values of the corresponding time intervals before and after lidocaine injection was significant throughout the 10 -min period (two-way repeated measures ANOVA and LSD test, $p<0.001$ ) (Fig. 1C).

Systemic arterial blood pressure of the animals was $87.6 \pm 1 \mathrm{mmHg}$ throughout the experiments. Neither meningeal application of $\mathrm{KCl}$ or phentolamine hydrochloride nor injection of lidocaine into the trigeminal ganglion caused significant changes in arterial blood pressure.

\subsection{Stimulation-induced changes in CGRP levels in CSF}

CSF taken immediately after opening the cisterna magna contained $245 \pm 25 \mathrm{pg} / \mathrm{ml} \mathrm{CGRP}$, a second sample 15 min later $133 \pm 20 \mathrm{pg} / \mathrm{ml}$. Around 15 min after craniotomy the CGRP concentrations in the CSF further decreased to $108 \pm 12 \mathrm{pg} / \mathrm{ml}$. The CGRP concentration of four following CSF samples collected at intervals of 15 min still tended to decrease but values were not significantly different compared to the previous measurement $(n=8-24$, repeated measures ANOVA and LSD post-hoc test, Fig. 2A). Thus, for the main experiments we started dural stimulation and CSF sampling only 30 min after completing the trepanation.

In samples taken after $\mathrm{KCl}$ application onto the dura mater the CGRP concentration was increased from $84.3 \pm 4.9 \mathrm{pg} / \mathrm{ml}$ to $94.9 \pm 6.1 \mathrm{pg} / \mathrm{ml}$, i.e. by $14.5 \pm 5.7 \%$ over baseline ( $n=18$, $t$-test, $p=0.043$, Fig. $2 \mathrm{~B}$ ). Injection of lidocaine into the trigeminal ganglion had no effect on CGRP concentrations in the CSF but abolished the CGRP releasing effect of meningeal $\mathrm{KCl}$ application (Fig. 2C). After ganglion anaesthesia in 8 experiments the CGRP concentration in the CSF following $\mathrm{KCl}$ application to the dura mater remained nearly at baseline ( $91 \pm 9.1 \%$ of baseline) with no significant change (Wilcoxon, $p=0.24$ ).

\subsection{Stimulation-induced changes in plasma levels of CGRP}

Basal levels of CGRP in the jugular venous plasma taken 10 min prior to stimulation of the dura mater with $60 \mathrm{mM} \mathrm{KCl}$ were $15.0 \pm 2.8 \mathrm{pg} / \mathrm{ml}$. In the sample taken after stimulation the CGRP concentration in the jugular plasma was increased to $21.8 \pm 2.8 \mathrm{pg} / \mathrm{ml}$ ( $148 \%$ of the basal concentration; Wilcoxon, $n=8, p=0.017$ ) (Fig. 2D).

\subsection{CGRP concentrations in the hemisected cranial preparation}

In the ex vivo hemisected rat skull preparation with adhering dura mater the basal release of CGRP measured in the incubation fluid was $15.6 \pm 4.7 \mathrm{pg} / \mathrm{ml}(n=8) . \mathrm{KCl}$ application at $60 \mathrm{mM}$ concentration increased the CGRP concentration to $76.7 \pm 14.9 \mathrm{pg} / \mathrm{ml}$ ( $492 \%$ of the baseline; Wilcoxon, $p<0.001$, Fig. 2E).

3.6. CGRP- and tyrosine hydroxylase-immunoreactive nerve fibers in the dura mater and medullary pia mater

In the parietal dura mater and medullary pia mater whole mount preparations CGRP-immunoreactive nerve fibers were seen running in small nerve bundles or as single axons, which formed branches and loose nerve plexuses around arterial blood vessels. In the dura mater 


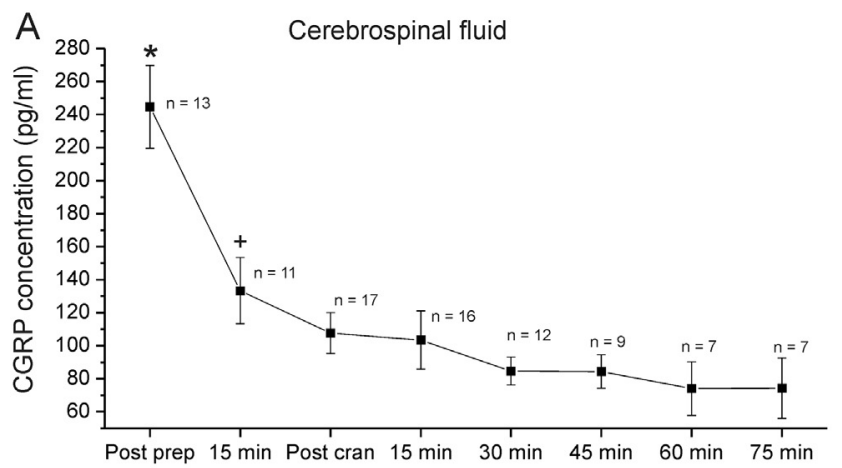

B Cerebrospinal fluid C CSF post lidocaine

Jugular plasma
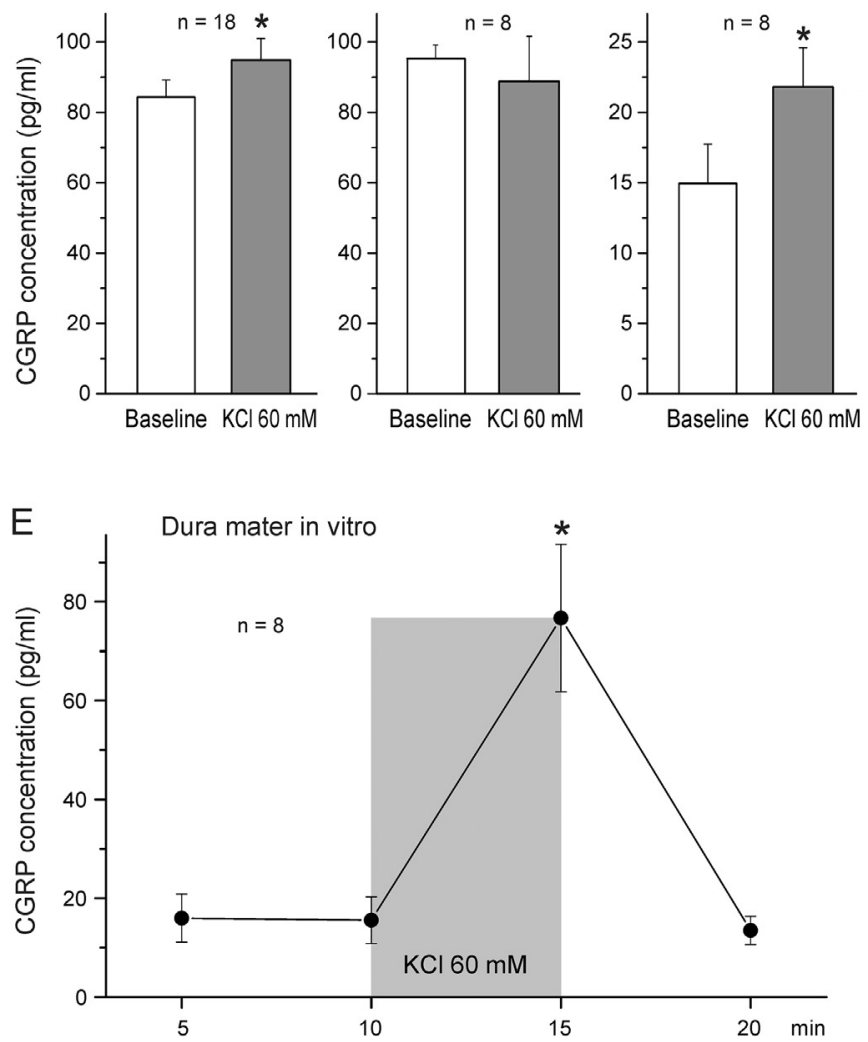

Fig. 2. CGRP concentrations measured in the cerebrospinal fluid (CSF) of the cisterna magna (A-C), the plasma of the jugular vein (D) and the SIF in the hemisected rat head lined with dura mater (E). A: Decrease in CGRP concentrations in the course of the surgery, immediately after opening of the cisterna magna (post prep) and 15 min later, immediately after the craniotomy (post cran) and at regular intervals of $15 \mathrm{~min}$; * different to all other measurements, ${ }^{+}$different only to the 60 min value (repeated measures ANOVA and LSD test). B: CGRP concentrations in the CSF before (Baseline) and after topical application of $\mathrm{KCl}(60 \mathrm{mM})$ onto the dura mater. C: Same as in B but after lidocaine injection into the trigeminal ganglion. D: CGRP levels in jugular plasma before and after stimulation of the dura mater with $\mathrm{KCl}(60 \mathrm{mM})$ compared to baseline. E: CGRP concentrations at baseline (5 and $10 \mathrm{~min})$ and after $\mathrm{KCl}(60 \mathrm{mM})$ administration. * Significant difference from baseline in B-E.

tyrosine hydroxylase-immunoreactive nerve fiber bundles were seen in close proximity to both CGRP-immunoreactive afferents and meningeal arteries (Fig. 3).

\section{Discussion}

Neurovascular changes induced by the release of vasoactive neuropeptides from trigeminal afferents into meningeal tissues can be used to indirectly assess changes in trigeminal afferent activity. Vasodilatory
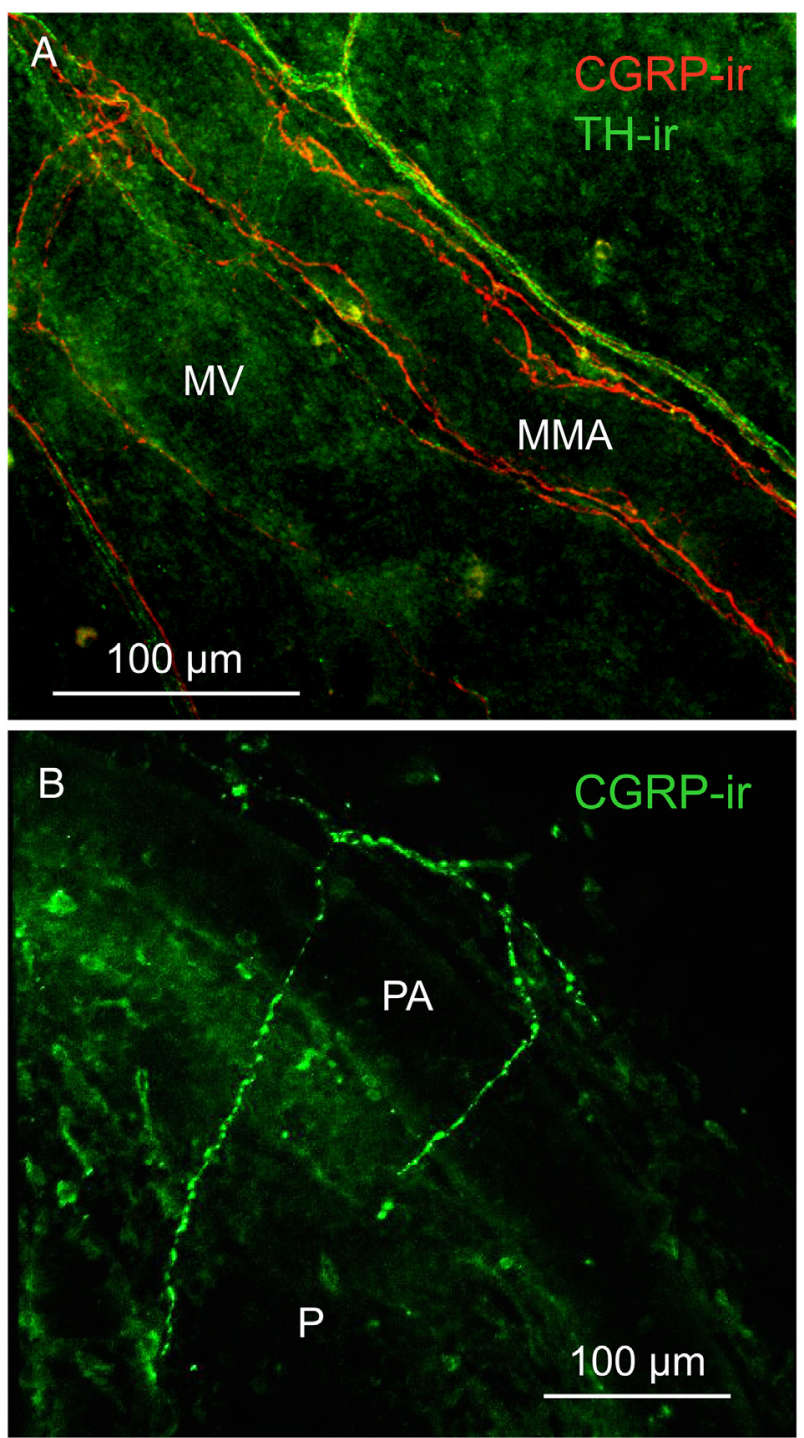

Fig. 3. Immunohistochemical staining of nerve fibers in whole mounts of the rat parietal dura mater (A) and the dorsal medullary pia mater (B). A: Nerve fibers immunoreactive to CGRP (red) and to tyrosine hydroxylase (green) in the dura accompany arterial blood vessels (MMA, branch of the middle meningeal artery). MV, meningeal venous vessel. $B$ : In the medullary pia mater an artery (PA) extending from the pial layer $(\mathrm{P})$ is innervated by a branched CGRP-immunoreactive fiber (green). B is composed of a stack of 9 images at a distance of $3 \mu \mathrm{m}$.

effects can be measured by video microscopy as calibre changes of meningeal arteries (Akerman et al., 2002; Petersen et al., 2004; Williamson et al., 1997) and by blood flow recordings using laser Doppler flowmetry (Carmody et al., 1996; Dux et al., 2003, 2007; Kurosawa et al., 1995). Vasoactive neuropeptides like substance P and CGRP released from trigeminal afferents have been quantified using radioimmunoassay (Durham et al., 2006; Flores et al., 2001; Garry and Hargreaves, 1992; Knight et al., 1999) and ELISA methods (Eberhardt et al., 2008; Ebersberger et al., 1999; Gupta et al., 2010; Messlinger et al., 1995; Sauer et al., 2001; Strecker et al., 2006). Here we report a new model combining both blood flow recordings in the dura mater and the medulla oblongata with the measurement of CGRP which is released from these tissues and drained into the jugular vein and the CSF. $\mathrm{KCl}$ at $60 \mathrm{mM}$ depolarizing meningeal nerve fibers was applied to the exposed cranial dura mater to induce CGRP release into the cranial circulation and the cerebrospinal fluid accompanied by increased medullary blood flow, which was abolished after local anaesthesia of the trigeminal ganglion. In the hemisected rat preparation a similar amount of 
CGRP is released by activation of $\mathrm{Ca}^{2+}$ conducting receptor channels such as transient receptor potential vanilloid 1 (TRPV1) through agonists like capsaicin at concentrations of 0.1-1 $\mu \mathrm{M}$ (Gupta et al., 2010). However, repetitive stimulation with capsaicin causes tachyphylaxis, and at concentrations of $\geq 1 \mu \mathrm{M}$ capsaicin decreases meningeal blood flow, most likely by a direct action on meningeal arteries (Dux et al., 2003). Thus $\mathrm{KCl}$ appeared advantageous for these types of experiments.

\subsection{CGRP release into blood and CSF from different sources}

The concentration of CGRP in the jugular blood was in the range of reported plasma concentrations found in the rat (Hoffmann et al., 2012) or pig (Arulmani et al., 2004b). In the cat and in humans jugular CGRP levels have been reported to be higher, which may be due to species differences (Goadsby and Edvinsson, 1993; Goadsby et al., 1988; Knight et al., 1999, 2001; Zagami et al., 1990).

The CGRP levels measured in the CSF collected from the cisterna magna are also in agreement with a study of Hoffmann et al. (2012) (Hoffmann et al., 2012). The basal levels are nearly one order of magnitude higher than in jugular plasma, raising questions about the sites from which CGRP is released and by which routes CGRP is drained. Since a blood-brain barrier is lacking in the dura mater, most of the CGRP released from meningeal afferent fibers is probably filtrated into capillaries and postcapillary venules (Fig. 4). The venous blood is drained via dural veins into the sagittal and transverse sinuses and eventually in the jugular vein. It is less likely that the CGRP released from dural afferents appears primarily in the CSF, because the cranial dura mater is isolated from the subarachnoidal space by the subdural neurothelium, the cells of which are connected by tight junctions (Rascol and Izard, 1976). Other investigations showed that a diffusion barrier between dura mater and the subarachnoidal space is mainly composed of a pial (outer arachnoid) layer beneath the dura (Feurer and Weller, 1991).

Pial arteries running through the subarachnoidal space are also innervated by CGRP-immunoreactive nerve fibers (Edvinsson et al., 1987; Fricke et al., 2001), and the same applies for blood vessels in the pia mater covering the medulla, as demonstrated by immunohistochemistry (see Figs. 3 and 4). CGRP released from these fibers is probably directly drained into the CSF; it is unlikely that much of this CGRP is filtrated into the cerebral blood vessels, where it must cross the bloodbrain barrier, which is functionally a blood-liquor barrier in this case. Whether and at which amount CGRP is able to penetrate this barrier in either direction is unknown. Small but measurable amounts of neuropeptides of a size comparable with that of CGRP have been reported to be taken up by the brain through the blood-brain barrier (reviewed in (Ermisch et al., 1985)); for ${ }^{3} \mathrm{H}$-labeled substance $\mathrm{P}$ this rate is about $0.5 \%$. Inversely, some CGRP may slowly move into the venous system together with the CSF absorbed through the arachnoidal (Pacchioni) granulations.

Regarding the relatively high CGRP concentrations found in the CSF compared to blood plasma, a significant proportion of CGRP may be released from the central terminals of trigeminal afferents in superficial layers of the spinal trigeminal nucleus (Lennerz et al., 2008), probably diffusing to the dorsal surface of the medulla oblongata and into the surrounding ventricular space (Fig. 4). This assumption is supported by the finding that anaesthesia of the trigeminal ganglion abolished the increase in CGRP levels in the CSF caused by meningeal $\mathrm{KCl}$ application. Using the microprobe techniques we have earlier shown that noxious stimulation of the dura mater by acidic solution caused considerable accumulation of substance $P$ in superficial layers of the spinal trigeminal nucleus (Schaible et al., 1997). In a newly designed preparation we found high amounts of CGRP released from medulla oblongata slices of mice after stimulation with capsaicin or $\mathrm{KCl}$ (Kageneck et al., 2014). Different to the blood plasma, CGRP can probably accumulate in the CSF because of the lack of peptidases (especially the neutral endopeptidase), which rapidly break down CGRP in the blood plasma.

\subsection{Vascular effects of released CGRP}

Regardless of looking at the venous plasma or the CSF, it is questionable if the low CGRP concentrations found in either compartment can cause significant vasodilatation. Isolated cerebral arteries of different species (cat, rabbit, rat) preconstricted with $\mathrm{PGF}_{2 \alpha}$ showed half-maximal relaxation $\left(\mathrm{EC}_{50}\right)$ with CGRP in the range of 5-10 nM (19$38 \mathrm{ng} / \mathrm{ml}$ ) (Edvinsson et al., 1985, 1987; McCulloch et al., 1986) and the $\mathrm{EC}_{50}$ for CGRP to relax rat intracerebral arterioles was $3.9 \mathrm{nM}$ (15 ng/ml) (Edwards et al., 1991) but in jugular plasma the CGRP concentration was not above $5.3 \mathrm{pM}(20 \mathrm{pg} / \mathrm{ml})$. Even CGRP at $25 \mathrm{pM}$ (95 pg/ml) concentration as found in the CSF during the steady state of sampling in our experiments may not cause relevant vasorelaxation. The CGRP concentrations at the blood vessels innervated by these terminals can be considered to be higher by several orders, which easily explains their potent vasodilatory capacity (see Figs. 3 and 4). Taken together, it is unlikely that the CGRP released into the CSF can cause a detectable increase in medullary blood flow, rather it is the direct vasodilatory effect of activated primary afferent terminals within the spinal trigeminal nucleus that drives an increased blood supply, possibly supplemented by the direct innervations of the pial medullary arteries as evident from immunohistochemistry.

\subsection{Neuronal and vascular effects of $\mathrm{KCl}$}

Topical application of depolarizing potassium concentrations caused a decrease in meningeal blood flow unless the dura mater was pretreated with phentolamine blocking alpha-adrenoceptors. This

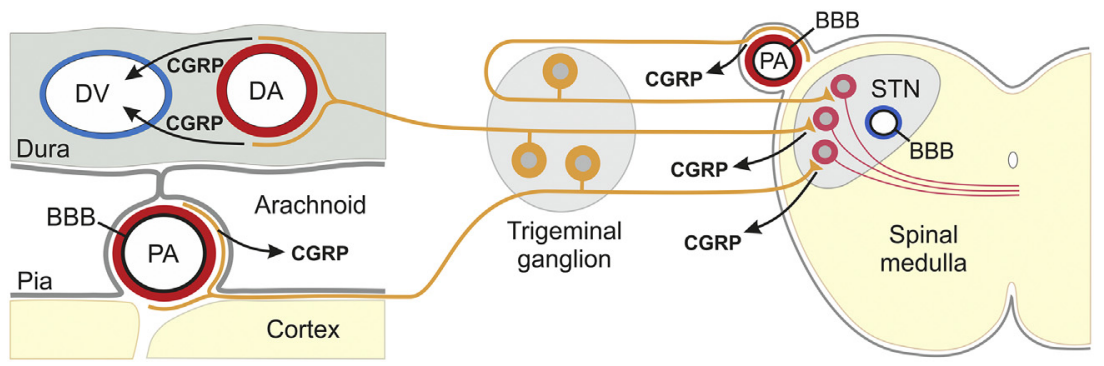

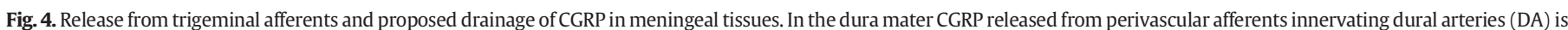

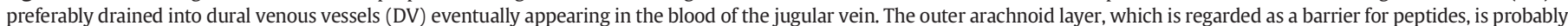

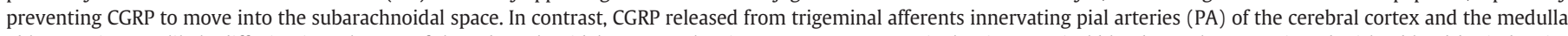

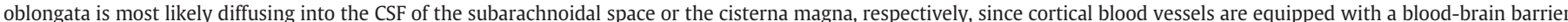

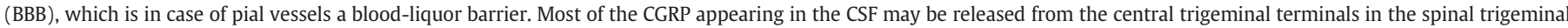

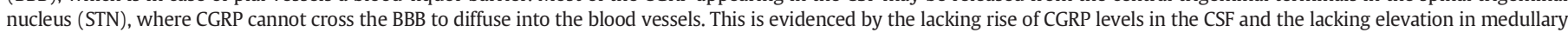
blood flow after anaesthesia of the trigeminal ganglion. 
indicates that $\mathrm{KCl}$ is able to depolarise also postganglionic sympathetic fibers, which richly supply dural arteries (Edvinsson and Uddman, 1981; Keller et al., 1989) and counteract the CGRP mediated vasodilatory effect upon the release of vasoconstrictory mediators like norepinephrine. Nevertheless, after blocking alpha-adrenoceptors, $\mathrm{KCl}$ still failed to increase the meningeal blood flow significantly, which may indicate further mechanisms counteracting the vasodilatory action of CGRP. In contrast, $\mathrm{KCl}$ application onto the dura caused a small but consistent increase in medullary blood flow. The vasodilatory effect of CGRP in meningeal and cerebral arteries as in several other arterial vessels has been shown to depend preferably on the activation of ATP-sensitive $\mathrm{K}^{+}$channels ( $\mathrm{K}_{\text {ATP }}$ ) and the subsequent hyperpolarisation of the vascular smooth muscle cells through $\mathrm{K}^{+}$outflow (Gozalov et al., 2005; Ko et al., 2008; Quayle et al., 1997). This potent mechanism seems to be compensated by direct vascular smooth muscle depolarization induced by $\mathrm{K}^{+}$resulting in a net effect of near zero arterial vasodilatation. Since at an extracellular concentration of $60 \mathrm{mM} \mathrm{K}^{+}$the reversal potential of $\mathrm{K}_{\mathrm{ATP}}$ channels is close to $-20 \mathrm{mV}$, voltage-sensitive $\mathrm{Ca}^{2+}$ channels are open and activate contraction of smooth vascular muscle cells (Nelson and Quayle, 1995). Phentolamine can antagonize the effect of released noradrenalin but not the direct depolarizing effect of $\mathrm{KCl}$.

$\mathrm{KCl}$ application to the cortical surface is an established method to induce cortical spreading depression, which is characterized by metabolic disturbance in superficial cortical areas accompanied by a transient increase in flow followed by long-lasting suppression of cortical functions (Ueda et al., 1997). In our experiments there was not any evidence for CSD-related changes in meningeal or medullary blood flow following $\mathrm{KCl}$ application to the dura mater.

\subsection{Effects of trigeminal ganglion anaesthesia}

Injection of lidocaine into the ipsilateral trigeminal ganglion abolished the increase in medullary blood flow evoked by local stimulation of the dura mater with $\mathrm{KCl}$. This treatment presumably blocked all the activity conducted through the ganglion. Anaesthesia of the trigeminal ganglion has previously been shown to reduce the ongoing activity of spinal trigeminal neurons with meningeal afferent input (Roch et al., 2007) indicating that a certain amount of afferent input is required to drive the activity of second order neurons. Thus it is likely that blood flow increases following dilatation of medullary blood vessels depend primarily on CGRP release from the central afferent terminals in the spinal trigeminal nucleus.

\subsection{Clinical implications}

The release of CGRP in the spinal trigeminal nucleus is of considerable relevance for the understanding of central nociceptive mechanisms involved in trigeminal nociception as the basic principle of headache and facial pain. Peripheral sensitization of trigeminal afferents leading to enhanced release of CGRP in the trigeminal nucleus is considered as the major peripheral pathophysiological mechanism involved in the generation of primary headaches. Moreover, CGRP is a modulator of nociceptive processing on the level of the dorsal horn and the spinal trigeminal nucleus (Storer et al., 2004; Sun et al., 2004; Yu et al., 2002). Our group and others have provided evidence that this central CGRPdriven neurotransmission is an important site for CGRP receptor inhibitors reducing pain in migraine attacks (Fischer et al., 2005; Sixt et al., 2009; Storer et al., 2004). CGRP is released upon direct stimulation of the human and cat trigeminal ganglion (Goadsby and Edvinsson, 1993; Goadsby et al., 1988) and during attacks of migraine and cluster headache (Goadsby and Edvinsson, 1994; Goadsby et al., 1990), i.e. during events likely to be associated with strong activation of meningeal nociceptors. This has been evidenced based on measurements of CGRP plasma levels either in the venous outflow from the head (Goadsby et al., 1990) or in peripheral veins (Goadsby et al., 1988). We can now speculate from our experiments that a considerable part of the CGRP released from activated trigeminal afferents during experimental conditions or severe attacks of primary headaches is drained into the CSF and does not appear in the venous system. Most of the CGRP in the CSF may be released from central terminals of meningeal afferents in the spinal trigeminal nuclear complex.

\section{Acknowledgements}

We like to thank Jana Schramm, Birgit Vogler, Annette Kuhn and Susanne Haux-Oertel for technical assistance. The project was supported by the Friedrich-Alexander-University Erlangen-Nürnberg (Emerging Fields Project: Medicinal Redox Inorganic Chemistry), the Humboldt Foundation (travel grant to MD), the FP7 project EUROHEADPAIN (Grant agreement no: 602633) and the K119597 project of the Hungarian National Research, Development and Innovation Office.

\section{References}

Akerman, S., Williamson, D.J., Kaube, H., Goadsby, P.J., 2002. The role of histamine in dural vessel dilation. Brain Res. 956, 96-102.

Amrutkar, D.V., Ploug, K.B., Olesen, J., Jansen-Olesen, I., 2011. Role for voltage gated calcium channels in calcitonin gene-related peptide release in the rat trigeminovascular system. Neuroscience 172, 510-517.

Andres, K.H., von Düring, M., Muszynski, K., Schmidt, R.F., 1987. Nerve fibres and their terminals of the dura mater encephali of the rat. Anat. Embryol. 175, 289-301.

Arulmani, U., Maassenvandenbrink, A., Villalón, C.M., Saxena, P.R., 2004a. Calcitonin generelated peptide and its role in migraine pathophysiology. Eur. J. Pharmacol. 500, 315-330.

Arulmani, U., Heiligers, J., Garrelds, I., Sánchez-López, A., Willems, E., Villalón, C., Saxena, P., 2004b. Effects of sumatriptan on capsaicin-induced carotid haemodynamic changes and CGRP release in anaesthetized pigs. Cephalalgia 24, 717-727.

Bellamy, J.L., Cady, R.K., Durham, P.L., 2006. Salivary levels of CGRP and VIP in rhinosinusitis and migraine patients. Headache 46, 24-33.

Bigal, M.E., Walter, S., Rapoport, A.M., 2015. Therapeutic antibodies against CGRP or its receptor. Br. J. Clin. Pharmacol. 79, 886-895.

Carmody, J., Pawlak, M., Messlinger, K., 1996. Lack of a role for substance P in the control of dural arterial flow. Exp. Brain Res. 111, 424-428.

Cernuda-Morollón, E., Larrosa, D., Ramón, C., Vega, J., Martínez-Camblor, P., Pascual, J., 2013. Interictal increase of CGRP levels in peripheral blood as a biomarker for chronic migraine. Neurology 81, 1191-1196.

Durham, P.L., Niemann, C., Cady, R., 2006. Repression of stimulated calcitonin gene-related peptide secretion by topiramate. Headache 46, 1291-1295.

Dux, M., Messlinger, K., 2001. Histological demonstration of increased vascular permeability in the dura mater of the rat. Microsc. Res. Tech. 53, 229-231.

Dux, M., Messlinger, K., 2015. New options for migraine treatment: focus on CGRP blocking antibodies. Drugs Future 40, 589-599.

Dux, M., Schwenger, N., Messlinger, K., 2002. Possible role of histamine (H1- and H2-) receptors in the regulation of meningeal blood flow. Br. J. Pharmacol. 137, 874-880.

Dux, M., Sántha, P., Jancsó, G., 2003. Capsaicin-sensitive neurogenic sensory vasodilatation in the dura mater of the rat. J. Physiol. 552, 859-867.

Dux, M., Rosta, J., Pintér, S., Sántha, P., Jancsó, G., 2007. Loss of capsaicin-induced meningeal neurogenic sensory vasodilatation in diabetic rats. Neuroscience 150, 194-201.

Eberhardt, M., Hoffmann, T., Sauer, S.K., Messlinger, K., Reeh, P.W., Fischer, M.J.M., 2008 Calcitonin gene-related peptide release from intact isolated dorsal root and trigeminal ganglia. Neuropeptides 42, 311-317.

Eberhardt, M., Neeb, L., Vogel, E.-M., Tiegs, G., Reuter, U., Messlinger, K., Fischer, M.J.M., 2009. Glyceroltrinitrate facilitates stimulated CGRP release but not gene expression of CGRP or its receptor components in rat trigeminal ganglia. Neuropeptides 43 , 483-489.

Ebersberger, A., Averbeck, B., Messlinger, K., Reeh, P.W., 1999. Release of substance P, calcitonin gene-related peptide and prostaglandin E2 from rat dura mater encephali following electrical and chemical stimulation in vitro. Neuroscience 89, 901-907.

Edvinsson, L., Goadsby, P.J., 1994. Neuropeptides in migraine and cluster headache. Cephalalgia Int. J. Headache 14, 320-327.

Edvinsson, L., Uddman, R., 1981. Adrenergic, cholinergic and peptidergic nerve fibres in dura mater-involvement in headache? Cephalalgia Int. J. Headache 1, 175-179.

Edvinsson, L., Fredholm, B.B., Hamel, E., Jansen, I., Verrecchia, C., 1985. Perivascular peptides relax cerebral arteries concomitant with stimulation of cyclic adenosine monophosphate accumulation or release of an endothelium-derived relaxing factor in the cat. Neurosci. Lett. 58, 213-217.

Edvinsson, L., Ekman, R., Jansen, I., McCulloch, J., Uddman, R, 1987. Calcitonin gene-related peptide and cerebral blood vessels: distribution and vasomotor effects. J. Cereb. Blood Flow Metab. 7, 720-728.

Edwards, R.M., Stack, E.J., Trizna, W., 1991. Calcitonin gene-related peptide stimulates adenylate cyclase and relaxes intracerebral arterioles. J. Pharmacol. Exp. Ther. 257, 1020-1024.

Ermisch, A., Rühle, H.J., Landgraf, R., Hess, J., 1985. Blood-brain barrier and peptides. J. Cereb. Blood Flow Metab. 5, 350-357. 
Escott, K.J., Connor, H.E., Brain, S.D., Beattie, D.T., 1995. The involvement of calcitonin gene-related peptide (CGRP) and substance $\mathrm{P}$ in feline pial artery diameter responses evoked by capsaicin. Neuropeptides 29, 129-135.

Feindel, W., Penfield, W., McNAUGHTON, F., 1960. The tentorial nerves and Iocalization of intracranial pain in man. Neurology $10,555-563$.

Feurer, D.J., Weller, R.O., 1991. Barrier functions of the leptomeninges: a study of normal meninges and meningiomas in tissue culture. Neuropathol. Appl. Neurobiol. 17, 391-405.

Fischer, M.J.M., Messlinger, K., 2007. Cannabinoid and vanilloid effects of R(+)methanandamide in the hemisected meningeal preparation. Cephalalgia Int. J. Headache 27, 422-428.

Fischer, M.J.M., Koulchitsky, S., Messlinger, K., 2005. The nonpeptide calcitonin gene-related peptide receptor antagonist BIBN4096BS lowers the activity of neurons with meningeal input in the rat spinal trigeminal nucleus. J. Neurosci. 25, 5877-5883.

Flores, C.M., Leong, A.S., Dussor, G.O., Harding-Rose, C., Hargreaves, K.M., Kilo, S., 2001. Capsaicin-evoked CGRP release from rat buccal mucosa: development of a model system for studying trigeminal mechanisms of neurogenic inflammation. Eur. J. Neurosci. 14, 1113-1120.

Fricke, B., Andres, K.H., Von Düring, M., 2001. Nerve fibers innervating the cranial and spinal meninges: morphology of nerve fiber terminals and their structural integration. Microsc. Res. Tech. 53, 96-105.

Garry, M.G., Hargreaves, K.M., 1992. Enhanced release of immunoreactive CGRP and substance P from spinal dorsal horn slices occurs during carrageenan inflammation. Brain Res. 582, 139-142.

Giamberardino, M.A., Affaitati, G., Curto, M., Negro, A., Costantini, R., Martelletti, P., 2016. Anti-CGRP monoclonal antibodies in migraine: current perspectives. Intern. Emerg. Med. 11, 1045-1057.

Goadsby, P.J., Edvinsson, L., 1993. The trigeminovascular system and migraine: studies characterizing cerebrovascular and neuropeptide changes seen in humans and cats. Ann. Neurol. 33, 48-56.

Goadsby, P.J., Edvinsson, L., 1994. Human in vivo evidence for trigeminovascular activation in cluster headache. Neuropeptide changes and effects of acute attacks therapies. Brain J. Neurol. 117 (Pt 3), 427-434.

Goadsby, P.J., Edvinsson, L., Ekman, R., 1988. Release of vasoactive peptides in the extracerebral circulation of humans and the cat during activation of the trigeminovascular system. Ann. Neurol. 23, 193-196.

Goadsby, P.J., Edvinsson, L., Ekman, R., 1990. Vasoactive peptide release in the extracerebral circulation of humans during migraine headache. Ann. Neurol. 28, 183-187.

Gozalov, A., Petersen, K.A., Mortensen, C., Jansen-Olesen, I., Klaerke, D., Olesen, J., 2005. Role of KATP channels in the regulation of rat dura and pia artery diameter. Cephalalgia Int. J. Headache 25, 249-260.

Gupta, S., Amrutkar, D.V., Mataji, A., Salmasi, H., Hay-Schmidt, A., Sheykhzade, M. Messlinger, K., Olesen, J., Jansen-Olesen, I., 2010. Evidence for CGRP re-uptake in rat dura mater encephali. Br. J. Pharmacol. 161, 1885-1898.

Ho, T.W., Mannix, L.K., Fan, X., Assaid, C., Furtek, C., Jones, C.J., Lines, C.R., Rapoport, A.M., MK-0974 Protocol 004 study group, 2008. Randomized controlled trial of an oral CGRP receptor antagonist, MK-0974, in acute treatment of migraine. Neurology 70, 1304-1312.

Hoffmann, J., Wecker, S., Neeb, L., Dirnagl, U., Reuter, U., 2012. Primary trigeminal afferents are the main source for stimulus-induced CGRP release into jugular vein blood and CSF. Cephalalgia Int. J. Headache 32, 659-667.

Jansen-Olesen, I., Mortensen, A., Edvinsson, L., 1996. Calcitonin gene-related peptide is released from capsaicin-sensitive nerve fibres and induces vasodilatation of human cerebral arteries concomitant with activation of adenylyl cyclase. Cephalalgia Int. J. Headache 16, 310-316.

Jansen-Olesen, I., Baun, M., Amrutkar, D.V., Ramachandran, R., Christophersen, D.V., Olesen, J., 2014. PACAP-38 but not VIP induces release of CGRP from trigeminal nucleus caudalis via a receptor distinct from the PAC1 receptor. Neuropeptides 48, 53-64.

Kageneck, C., Nixdorf-Bergweiler, B.E., Messlinger, K., Fischer, M.J., 2014. Release of CGRP from mouse brainstem slices indicates central inhibitory effect of triptans and kynurenate. J. Headache Pain 15, 7.

Keller, J.T., Marfurt, C.F., 1991. Peptidergic and serotoninergic innervation of the rat dura mater. J. Comp. Neurol. 309, 515-534.

Keller, J.T., Marfurt, C.F., Dimlich, R.V., Tierney, B.E., 1989. Sympathetic innervation of the supratentorial dura mater of the rat. J. Comp. Neurol. 290, 310-321.

Knight, Y.E., Edvinsson, L., Goadsby, P.J., 1999. Blockade of calcitonin gene-related peptide release after superior sagittal sinus stimulation in cat: a comparison of avitriptan and CP122,288. Neuropeptides 33, 41-46.

Knight, Y.E., Edvinsson, L., Goadsby, P.J., 2001. 4991 W93 inhibits release of calcitonin gene-related peptide in the cat but only at doses with $5 \mathrm{HT}(1 \mathrm{~B} / 1 \mathrm{D})$ receptor agonist activity? Neuropharmacology 40, 520-525.

Ko, E.A., Han, J., Jung, I.D., Park, W.S., 2008. Physiological roles of K+ channels in vascular smooth muscle cells. J. Smooth Muscle Res. 44, 65-81.

Kurosawa, M., Messlinger, K., Pawlak, M., Schmidt, R.F., 1995. Increase of meningeal blood flow after electrical stimulation of rat dura mater encephali: mediation by calcitonin gene-related peptide. Br. J. Pharmacol. 114, 1397-1402.

Lassen, L.H., Haderslev, P.A., Jacobsen, V.B., Iversen, H.K., Sperling, B., Olesen, J., 2002. CGRP may play a causative role in migraine. Cephalalgia Int. J. Headache 22, 54-61.

Lennerz, J.K., Rühle, V., Ceppa, E.P., Neuhuber, W.L., Bunnett, N.W., Grady, E.F., Messlinger, K., 2008. Calcitonin receptor-like receptor (CLR), receptor activity-modifying protein 1 (RAMP1), and calcitonin gene-related peptide (CGRP) immunoreactivity in the rat trigeminovascular system: differences between peripheral and central CGRP receptor distribution. J. Comp. Neurol. 507, 1277-1299.
McCulloch, J., Uddman, R., Kingman, T.A., Edvinsson, L., 1986. Calcitonin gene-related peptide: functional role in cerebrovascular regulation. Proc. Natl. Acad. Sci. U. S. A. 83, 5731-5735

Meng, J., Ovsepian, S.V., Wang, J., Pickering, M., Sasse, A., Aoki, K.R., Lawrence, G.W., Dolly, J.O., 2009. Activation of TRPV1 mediates calcitonin gene-related peptide release, which excites trigeminal sensory neurons and is attenuated by a retargeted botulinum toxin with anti-nociceptive potential. J. Neurosci. 29, 4981-4992.

Messlinger, K., Hanesch, U., Kurosawa, M., Pawlak, M., Schmidt, R.F., 1995. Calcitonin gen related peptide released from dural nerve fibers mediates increase of meningeal blood flow in the rat. Can. J. Physiol. Pharmacol. 73, 1020-1024.

Messlinger, K., Hotta, H., Pawlak, M., Schmidt, R.F., 1997. Effects of the 5-HT1 receptor agonists, sumatriptan and CP 93,129, on dural arterial flow in the rat. Eur. J. Pharmacol. 332, 173-181.

Nelson, M.T., Quayle, J.M., 1995. Physiological roles and properties of potassium channels in arterial smooth muscle. Am. J. Phys. 268, C799-C822.

O'Connor, T.P., van der Kooy, D., 1988. Enrichment of a vasoactive neuropeptide (calcitonin gene related peptide) in the trigeminal sensory projection to the intracranial arteries. J. Neurosci. 8, 2468-2476.

Offenhauser, N., Zinck, T., Hoffmann, J., Schiemann, K., Schuh-Hofer, S., Rohde, W., Arnold G., Dirnagl, U., Jansen-Olesen, I., Reuter, U., 2005. CGRP release and c-fos expression within trigeminal nucleus caudalis of the rat following glyceryltrinitrate infusion. Cephalalgia Int. J. Headache 25, 225-236.

Olesen, J., Diener, H.-C., Husstedt, I.W., Goadsby, P.J., Hall, D., Meier, U., Pollentier, S., Lesko, L.M., BIBN 4096 BS Clinical Proof of Concept Study Group, 2004. Calcitonin gene-related peptide receptor antagonist BIBN 4096 BS for the acute treatment of migraine. N. Engl. J. Med. 350, 1104-1110.

Petersen, K.A., Birk, S., Doods, H., Edvinsson, L., Olesen, J., 2004. Inhibitory effect of BIBN4096BS on cephalic vasodilatation induced by CGRP or transcranial electrical stimulation in the rat. Br. J. Pharmacol. 143, 697-704.

Petersen, K.A., Lassen, L.H., Birk, S., Lesko, L., Olesen, J., 2005. BIBN4096BS antagonizes human alpha-calcitonin gene related peptide-induced headache and extracerebral artery dilatation. Clin. Pharmacol. Ther. 77, 202-213.

Quayle, J.M., Nelson, M.T., Standen, N.B., 1997. ATP-sensitive and inwardly rectifying potassium channels in smooth muscle. Physiol. Rev. 77, 1165-1232.

Rascol, M.M., Izard, J.Y., 1976. The subdural neurothelium of the cranial meninges in man. Anat. Rec. 186, 429-436

Ray, B.S., Wolff, H.G., 1940. Experimental studies on headache: pain sensitive structures of the head and their significance in headache. Arch. Surg. 1, 813-856.

Roch, M., Messlinger, K., Kulchitsky, V., Tichonovich, O., Azev, O., Koulchitsky, S., 2007. Ongoing activity in trigeminal wide-dynamic range neurons is driven from the periphery. Neuroscience 150, 681-691.

Sauer, S.K., Reeh, P.W., Bove, G.M., 2001. Noxious heat-induced CGRP release from rat sciatic nerve axons in vitro. Eur. J. Neurosci. 14, 1203-1208.

Schaible, H.G., Ebersberger, A., Peppel, P., Beck, U., Messlinger, K., 1997. Release of immunoreactive substance $P$ in the trigeminal brain stem nuclear complex evoked by chemical stimulation of the nasal mucosa and the dura mater encephali-a study with antibody microprobes. Neuroscience 76, 273-284.

Schuster, N.M., Rapoport, A.M., 2016. New strategies for the treatment and prevention of primary headache disorders. Nat. Rev. Neurol. 12, 635-650.

Sixt, M.-L., Messlinger, K., Fischer, M.J.M., 2009. Calcitonin gene-related peptide receptor antagonist olcegepant acts in the spinal trigeminal nucleus. Brain J. Neurol. 132, 3134-3141.

Steiger, H.J., Meakin, C.J., 1984. The meningeal representation in the trigeminal ganglion-an experimental study in the cat. Headache 24, 305-309.

Storer, R.J., Akerman, S., Goadsby, P.J., 2004. Calcitonin gene-related peptide (CGRP) modulates nociceptive trigeminovascular transmission in the cat. Br. J. Pharmacol. 142 1171-1181.

Strecker, T., Reeh, P.W., Weyand, M., Messlinger, K., 2006. Release of calcitonin gene-related peptide from the isolated mouse heart: methodological validation of a new model. Neuropeptides 40, 107-113.

Sun, R.-Q., Tu, Y.-J., Lawand, N.B. Yan, J.-Y, Lin, Q, Willis, W.D., 2004 Calcitonin gene-related peptide receptor activation produces PKA- and PKC-dependent mechanical hyperalgesia and central sensitization. J. Neurophysiol. 92, 2859-2866.

Tröltzsch, M., Denekas, T., Messlinger, K., 2007. The calcitonin gene-related peptide (CGRP) receptor antagonist BIBN4096BS reduces neurogenic increases in dural blood flow. Eur. J. Pharmacol. 562, 103-110.

Ueda, M., Watanabe, N., Ushikubo, Y., Tsuzuki, T., Aoki, K., Yamazaki, Y., Samejima, H., 1997. Changes in regional cortical temperature and cerebral blood flow after cortical spreading depression. Neurol. Med. Chir. (Tokyo) 37 (441-445-446).

Will, C., Messlinger, K., Fischer, M.J.M., 2016. Vessel diameter measurements at the medullary brainstem in vivo as an index of trigeminal activity. Brain Res. 1632, 51-57.

Williamson, D.J., Hargreaves, R.J., Hill, R.G., Shepheard, S.L., 1997. Intravital microscope studies on the effects of neurokinin agonists and calcitonin gene-related peptide on dural vessel diameter in the anaesthetized rat. Cephalalgia Int. J. Headache 17 518-524.

Yu, Y., Lundeberg, T., Yu, L.C., 2002. Role of calcitonin gene-related peptide and its antagonist on the evoked discharge frequency of wide dynamic range neurons in the dorsal horn of the spinal cord in rats. Regul. Pept. 103, 23-27.

Zagami, A.S., Goadsby, P.J., Edvinsson, L., 1990. Stimulation of the superior sagittal sinus in the cat causes release of vasoactive peptides. Neuropeptides 16, 69-75. 